\title{
How fats we eat modulate our immunity? ${ }^{\text {is }}$
}

\author{
Benjamin Buaud* \\ ITERG - Nutrition-Health and Lipid Biochemistry Team, 11 rue Gaspard Monge-ZA Pessac-Canéjan, 33610 Canéjan, France
}

Received 20 August 2019 - Accepted 10 January 2020

\begin{abstract}
The development and optimal functioning of our immune system is directly influenced by our diet. Any deficiency or excess of certain nutrients can affect the number and activity of immune cells. Among the nutrients identified, dietary fatty acids are described as having major effects on immunity. Indeed, the fatty acid composition of the membranes of immune cells seems to be easily modulated under the effect of dietary fats and the resulting rapid changes in composition are likely to generate functional effects on the reactivity and functioning of these cells within a very short period of time. Among the different mechanisms identified to explain the impact of dietary fatty acids on the immune function, the synthesis of lipid mediators from polyunsaturated fatty acids is a key one notably in the context of inflammation.
\end{abstract}

Keywords: n-3 PUFA / n-6 PUFA / immunity / diet / lipid mediators

\begin{abstract}
Résumé - Comment les graisses que nous consommons modulent-elles notre immunité ? Le développement et le fonctionnement optimal de notre système immunitaire sont directement influencés par notre alimentation. Toute carence ou excès de certains nutriments peut affecter le nombre et l'activité des cellules immunitaires. Parmi les nutriments identifiés, les acides gras alimentaires sont décrits comme ayant des effets majeurs sur l'immunité. En effet, la composition en acides gras des membranes des cellules immunitaires semble être facilement modulée sous l'effet des graisses alimentaires et les changements rapides de composition qui en résultent sont susceptibles de générer des effets fonctionnels sur la réactivité et le fonctionnement de ces cellules dans un délai très court. Parmi les différents mécanismes identifiés pour expliquer l'impact des acides gras alimentaires sur la fonction immunitaire, la synthèse de médiateurs lipidiques à partir d'acides gras polyinsaturés est un mécanisme clé, notamment dans le contexte de l'inflammation.
\end{abstract}

Mots clés : AGPI n-3 / AGPI n-6 / immunité / régime alimentaire / médiateurs lipidiques

\section{Introduction}

The immune system brings together all the resources that enable to protect the body against threatening agents or situations, both internal and external to the organism.

The immune function is influenced by many factors, including genetics, sex, early life events, age, hormonal status, stress... The nutritional status of the host is one of these modulating factors.

Adequate supply and balance of dietary nutrients are required for proper efficiency of the immune system, its development, maintenance, and functioning. Adequate intakes of micro- and macronutrients are therefore important in our Western societies, which are more characterized by a nonadequate modern dietary pattern: too much saturated fatty

\footnotetext{
Contribution to the Topical Issue "Lipids and health / Lipides et santé". *Correspondence: b. buaud@iterg. com
}

acids, salt, and sugar; low levels of n-3 fatty acids (Myles, 2014; Maggini et al., 2018). Among the macronutrients of interest, dietary lipids are described as having major effects on our immune system.

If the link between our diet and the development and optimal functioning of our immune system has been the subject of research for many years, the impact of dietary fat on immunity, on the other hand, has been investigated since the end of the 1970 s as reminded by Boissonneault (2008). From the first review in 1978, discussing fatty acids and immunity, many others followed dealing with the in vitro and in vivo evidence suggesting that dietary lipids play a role in modulating immune function.

It was then suggested that the observed effects involved modifications of the physical properties of the immune cell membrane, any modification of membrane order, also called fluidity, may have consequences of disrupting membrane processes, which are essential for the immune cells' response to any activation of the immune system. 

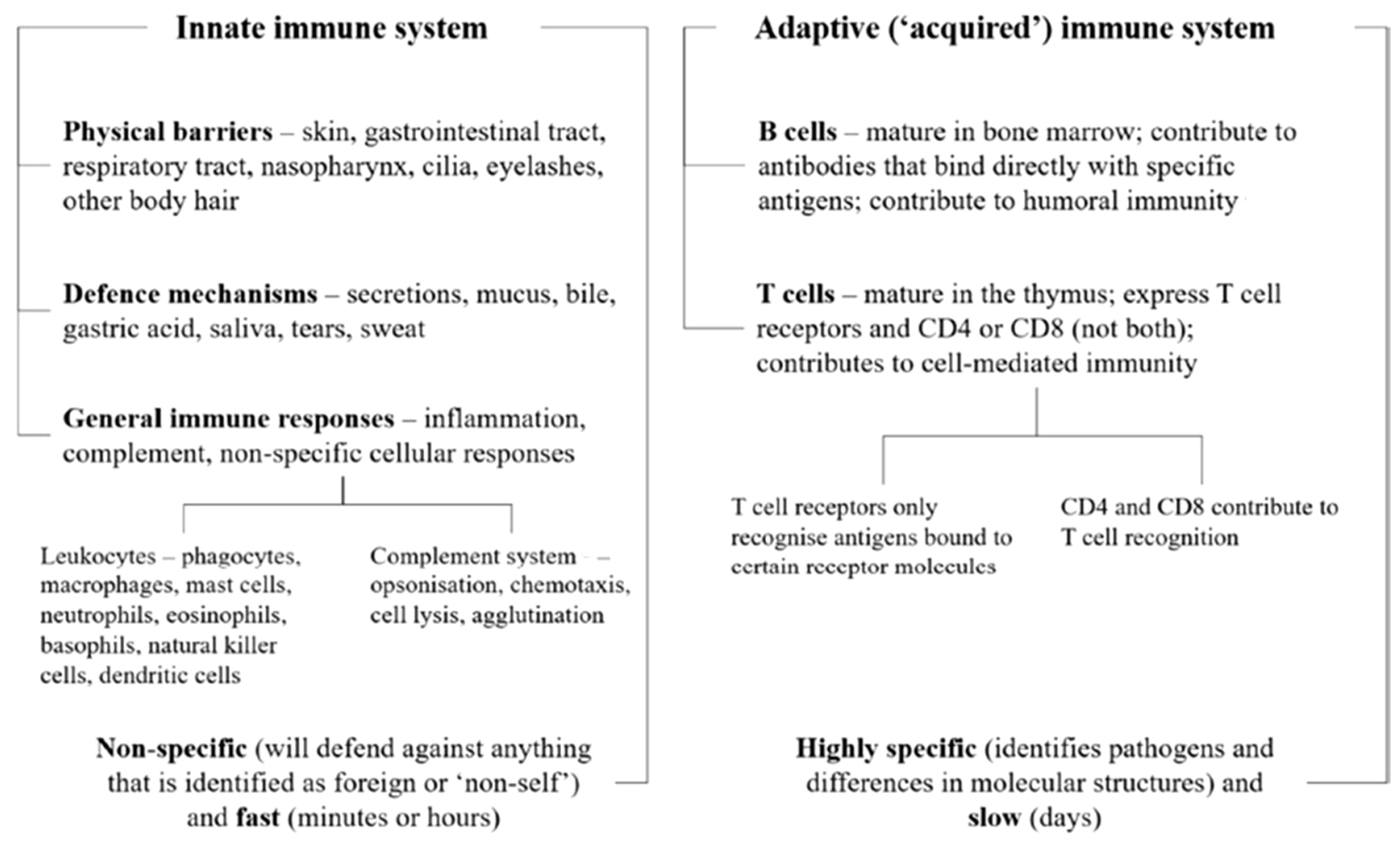

Fig. 1. Overview of the innate and adaptive immune systems. Modified from Maggini et al. (2018) with permission from MDPI.

The subsequent discovery that eicosanoids, biologically active lipid mediators produced from 20 carbon polyunsaturated fatty acids (PUFA), notably from arachidonic acid (ARA, $20: 4 n-6$ ), can play a role in inflammation (a component of the immune response) and in regulating immune cell functions, has led to research on the effects of ARA. The observations made highlighted the possibility that the effects of dietary fatty acids on immunity could be explained by a change in the production of these lipid mediators (Kinsella et al., 1990; Calder et al., 1992; Calder, 2013).

The more recent discovery of other bioactive lipid mediators from n-3 PUFA and known as specialized proresolution lipid mediators derived because of their role as key signaling molecules in the resolution of inflammation suggest that these lipid mediators appear to have beneficial effects on multiple modalities of immune function (Calder, 2017; Gilroy and Bishop-Bailey, 2019).

The purpose of this article is to provide a non-exhaustive overview of the mechanisms known to date to explain how fats we eat, in particular dietary n-6 and n-3 PUFA, can influence how our immune system works.

\section{General data on the immune system}

The immune response acts to protect the host from infectious agents that exist in the environment, such as microbes (viruses, bacteria, fungi...), and from other insults such as abnormalities of host tissues (cancer), and tissue trauma. Under certain conditions, the immune system can itself cause disease by involuntarily responding against host tissues as in cases of autoimmunity or contribute to the etiology of other diseases (atherosclerosis, psoriasis).

As summarized by Maggini et al. (2018), the immune system utilizes three distinct layers according to the nature of the threat. The first one includes physical and biochemical barriers, such as skin, epithelial lining of the gastrointestinal and respiratory tracts, and such as secretions, mucus and gastric acids, respectively. The second layer includes numerous different immune cells spread throughout the body such as monocytes, granulocytes, lymphocytes, $\mathrm{T}$ and $\mathrm{B}$ cells. The third layer corresponds to the antibodies or immunoglobulins.

There are two types of immunity (Fig. 1). Innate immunity acts as the first line of defense and involves the physical and biochemical barriers associated with an unspecific cellular response mediated mainly by monocytes, neutrophils, natural killer (NK) cells and dendritic cells. All work together to fight off pathogens before they can start an active infection. Acquired (adaptive) immunity acts as a second line of defense by providing long-term protection against particular pathogens through the production of antibodies specific to them. The acquired immune response involves $\mathrm{T}$ lymphocytes that will release chemical mediators (cytokines) (cell-mediated immunity), as well as B lymphocytes that will produce antibodies that contribute to the recognition and elimination of pathogens (humoral immunity). When the immune response is brought into play, both types of immunity are likely to occur (Hubler and Kennedy, 2016).

To quote Maggini et al. (2018), "optimal immune function is dependent on a healthy immune system", an adequate nutrition is essential to ensure all the needs required for the 
development, maintenance and expression of the immune system. To guarantee the latter, dietary fats are among the macronutrients having crucial roles on the immune system.

\section{Dietary fats: what are we talking about?}

Dietary fats we consume are mainly triglycerides, which are the main form of lipids in commonly occurring oils and fats.

Triglycerides are composed of three fatty acids esterified to a glycerol backbone. Dietary fatty acids are important sources of energy, major constituents of all cell membranes, key components involved in the metabolic, structural and functional pathways within the body, and precursors to signalling molecules. Dietary fatty acids can be saturated (SFA), monounsaturated (MUFA), or polyunsaturated (PUFA).

Concerning the latter, n-3 and n-6 PUFA are important components of the human's diet. The respective precursors of each family are the alpha-linolenic acid (ALA, 18:3n-3) and the linoleic acid (LA, 18:2n-6). Both are of plant origin. LA and ALA are described as essential fatty acids as they cannot be synthesized in higher animals including humans; they must be provided by the diet. Rich dietary sources of LA include many vegetable oils, such as sunflower, safflower, corn, and soybean oils. ALA is found in green plant tissues, in some common vegetable oils, including soybean and rapeseed oils, in some nuts (e.g. walnuts), and in flaxseeds and flaxseed oil.

In the body, LA and ALA are the substrates for the synthesis of very-long chain PUFA. By an analogous set of reactions catalyzed by the same enzymes, ALA can be converted to eicosapentaenoic (EPA, 20:5n-3) and docosahexaenoic acids (DHA, 22:6n-3), while LA can be converted to ARA. In humans, evidence suggests that the conversion of ALA into EPA and DHA may be limited (Burdge and Calder, 2006), partly due to the body's use of ALA and pathway intermediates as energy sources, and partly due to the inefficiency of the enzymes themselves. Estimates vary, but only a small proportion of ALA is converted to EPA ( 8 to $21 \%$ ) and especially to DHA (0.05 to 9\%) (Burdge and Wootton, 2002; Arterburn et al., 2006; Harnack et al., 2009; Blanchard et al., 2013). For this reason, the direct consumption of EPA and DHA is more effective for increasing their concentration in the body than is the consumption of ALA. The primary source of EPA and DHA is seafood, especially oily fish (tuna, salmon, mackerel, herring, and sardine). Dietary sources of ARA are meat, poultry, eggs, and organ meats.

N-6 and n-3 PUFA play vital roles in human health from conception onwards through every stage of human development, maturation and aging. However, most Western diets are described as generally containing an excess of n-6 PUFA, notably LA in the USA, over n-3 PUFA, particularly lacking in ALA, EPA and DHA (Baker et al., 2016; Sioen et al., 2017).

Diets rich in n-6 PUFAs are described as increasing the risk of development of inflammatory diseases, such as rheumatoid arthritis, inflammatory bowel disease, and asthma (Wall et al., 2010). On the other hand, epidemiological studies and several randomised control trials demonstrate positive effects of the consumption of EPA and DHA on hypertension, diabetes, reduction in cardiovascular disease morbidity and mortality, better visual and neurological development, and improvements in inflammatory disorders (Simopoulos, 2011; Baker et al., 2016).

\section{Dietary fatty acids as main components of the immune cell membranes}

It is admitted that dietary fatty acids can influence cellular responses and cell function of the immune cells by modulating the fatty acid composition of their membrane phospholipids.

As explained by Innes and Calder (2018), the fatty acid makeup of membrane phospholipids influences membrane order and lipid raft assembly, many second messengers being derived from membrane phospholipids, and the fatty acid composition of these messengers affecting their biological activity and potency. Another major point is that some lipid mediators are formed from fatty acids released from membrane phospholipids upon cellular activation.

\subsection{Fatty acid composition of the immune cell membranes}

To date, the fatty acid composition of immune cell membranes is relatively well described. Data obtained in human and rodents have shown that membrane phospholipids from different immune cells ( $\mathrm{T}$ and $\mathrm{B}$ lymphocytes, monocytes, neutrophils) contain mainly ARA, which alone represents about 15 to $25 \%$ of the total fatty acids contained in the membranes. The exact proportion of ARA depends of the cell type and the lipid fraction examined. The proportion of LA is between 6 and 10\%. In contrast, EPA and DHA, n-3 long chain PUFA derived from ALA, are minor constituents (Calder et al., 1990, 1994): ALA is rare $(\leq 0.1 \%$ of total fatty acids $)$, and EPA and DHA account for about 0.1 to $0.8 \%$ and 2 to $4 \%$ of total fatty acids, respectively (Kew et al., 2003a; Miles et al., 2004; Calder, 2015).

\subsection{Dietary fats as modulators of this composition}

Overall, it appears that dietary fats influence the fatty acid composition of membrane phospholipids in numerous studies, principally by increasing the amounts of the provided fatty acids. The consumption of certain fatty acids not only modifies their proportion in the lipids of the membranes of immune cells but may also have an impact on those of other fatty acids. This is the case, for example, of the ARA in the case of an $\mathrm{EPA}+\mathrm{DHA}$ contribution.

Thus, it has been shown that the enrichment of the diet with a given fatty acid increases the content of this fatty acid in the membranes of immune cells. Thus, in rodents, a diet rich in ARA, as well as a diet rich in EPA and DHA, leads to the respective enrichment in ARA and EPA and DHA of membranes of different cellular types (lymphocytes, macrophages and neutrophils) (Calder, 2015). Marshall and Johnston (1983) reported the fatty acid changes induced by feeding fats containing high ALA to LA ratio after a 2-month feeding period on the fatty acid composition of peripheral blood lymphocyte, thymocyte or splenocyte of rats. They observed that the n-3 PUFA reciprocally replaced the n-6 PUFA when 
higher ratios of ALA to LA were fed, the magnitude of fatty acid change increasing as the ALA/LA ratio increased. Tiwari et al. (1986) assessed the LA proportion in the membrane of splenocytes from mice fed with diets high or low in LA for 1, 4 or 6 weeks. They observed that the higher the LA level in the diet, the higher the level in the splenocyte membranes. The same authors observed that these changes in the fatty acid composition of splenocyte membranes were reflected in the fatty acid composition of $\mathrm{B}$ and $\mathrm{T}$ cell membranes (Tiwari et al., 1987).

In healthy elderly subjects, an enrichment of the ARA diet induces an increase in the proportion of ARA in blood mononuclear cells (Thies et al., 2001a), while an intake of ALA, in the form of flax oil, induces an increase in the EPA content of mononuclear cells and neutrophils (Kelley et al., 1993; Mantzioris et al., 1994; Kew et al., 2003b).

More studies have tested the effects of a diet enriched with fish oil or purified sources of EPA and DHA in healthy subjects. Consumption of such diets is accompanied by an increase in the EPA and DHA content of blood immune cells, which is dose-dependent (significant after 1 day of fish oil nutritional supplementation and maximum after 7 days), and at the expense of n-6 PUFA, such as ARA (Yaqoob et al., 2000; Thies et al., 2001a; Miles et al., 2004; Faber et al., 2011).

In his article on the relationship between n-3 PUFA, inflammation and immunity, Calder (2001) reviewed human studies having assessed the impact of n-3 PUFA supplementation on the ARA, EPA and DHA contents in the main membrane lipid fractions (total lipids, phospholipid, or individual phospholipids) of different immune cell types such as neutrophils, monocytes, $\mathrm{T}$ and $\mathrm{B}$ lymphocytes.

A dietary supply in EPA + DHA, which ranges from 0.3 to $14.4 \mathrm{~g} \mathrm{EPA}+\mathrm{DHA} /$ day for 2 to 12 weeks, induces an enrichment in n-3 PUFA in the membranes of the different cell types, at the expense of the ARA, whose proportion is reduced. The observed modifications are of different amplitudes, depending on the dose of EPA + DHA provided, the duration of administration, the cell type and the lipid fraction under observation. Slight variations are observed in neutrophils from the smallest amounts of EPA + DHA administered $(-8 \%$ for ARA, $+12 \%$ for DHA with EPA unchanged after 12 weeks of supplementation). On the other hand, the increased EPA and DHA proportions observed in neutrophil phospholipids in a dose-dependent manner after 4 weeks of supplementation with $2.25 \mathrm{~g} \mathrm{EPA}+\mathrm{DHA} /$ day remain unchanged beyond this time of 4 weeks.

Overall, it is generally accepted that the fatty acids in our diet contribute to the physical and functional properties of membranes and their representativeness is a major determinant of membrane fluidity. In addition to their role as membrane components, dietary fatty acids also act as substrates for the formation of bioactive oxygen derivatives.

\section{Polyunsaturated fatty acids, as precursors of major lipid mediators}

Maskrey et al. (2013), Duffney et al. (2018), and Gilroy and Bishop-Bailey (2019) recently published very comprehensive reviews about the different lipid mediators involved in immune regulation and inflammation resolution.

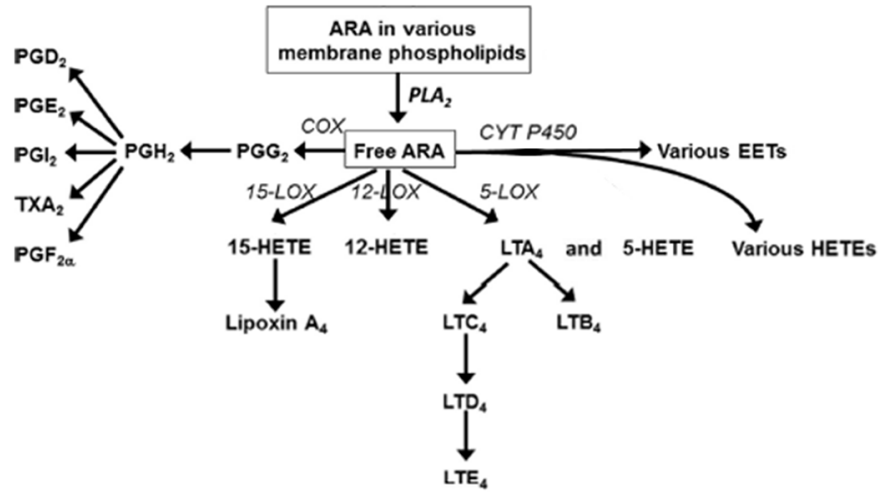

Fig. 2. Simplified overview of the pathways of eicosanoid synthesis from ARA. ARA: arachidonic acid; COX: cyclooxygenase; CYT P450: cytochrome P450 enzymes; EET: epoxyeicosatrienoic acid; HETE: hydroxyeicosatetraenoic acid; LOX: lipoxygenase; LT: leukotriene; PLA2: phospholipase A2; PG: prostaglandin; TX: thromboxane. Reprinted with modifications from Innes and Calder (2018) with permission from Elsevier.

As they wrote, lipids are important signalling molecules that regulate an array of immune responses, such as vascular hyper reactivity, pain, leukocyte trafficking and clearance, socalled resolution. Thus, they present in their respective reviews the major pathways involved in the biosynthesis of these mediators as well as their roles in inflammation and resolution. For more details, readers are invited to refer directly to their respective reviews.

In a very simplified way, lipid mediators are mainly synthesized from ARA for the n- 6 series, and from EPA and DHA for the n-3 series. Their biosynthetic pathways involve common enzymes, and are at the origin of a set of different molecules.

While DHA and EPA are upstream molecules for proresolving lipid mediator synthesis (described below), ARA is primarily further metabolized into eicosanoids, which are recognized as inflammatory lipid mediators.

Indeed, it is generally accepted that lipid mediators from n6 PUFA are considered to be rather pro-inflammatory, while those from n-3 PUFA are rather anti-inflammatory, the overall effect of these n-3 metabolites being to dampen inflammation and immune responses. There are, however, some exceptions to this statement.

\subsection{ARA as precursor of eicosanoids}

ARA is by far the most widely studied PUFA in the context of lipid mediator synthesis. ARA, once released from cell membrane phospholipids, is a substrate for cyclooxygenase (COX), lipoxygenase (LOX) and cytochrome P450 enzymes (CYT P450) to yield the eicosanoid family of mediators, which are important pro-inflammatory mediators in inflammatory response and inhibit immune function (Lewis et al., 1990; Tilley et al., 2001; Kalinski, 2012) (Fig. 2).

The first well-described lipid mediators are 2-series prostaglandins (PG) and thromboxans (TX), synthesized from ARA through the action of COX. 
Data concerning the most common PG, which are PGE2 and PGI2, indicate roles in enhancing vasodilatation, oedema formation and vascular permeability.

More precisely, as mention by Kalinski (2012), PGE2 is described as regulating multiple aspects of inflammation and multiple functions of different immune cells as presented PGE2 is a mediator of active inflammation, promoting local vasodilatation and local attraction and activation of neutrophils, macrophages, and mast cells at early stages of inflammation. In addition, PGE2 exhibits immunosuppressive and anti-inflammatory properties, by suppressing lymphocyte proliferation and natural killer cell activity and by inhibiting production of tumour necrosis factor (TNF)-alpha, interleukin (IL)-1, IL-6, IL-2 and interferon (IFN)-gamma.

Even the majority of these eicosanoids are considered as being pro-inflammatory, some of them may be trigger immunesuppressive effects in specific situations as explained by Gilroy and Bishop-Bailey (2019) (such as inhibition of the production of pro-inflammatory mediators, reduction of the ability of inflammatory leukocytes to phagocytose and kill microorganisms).

ARA can also be converted by LOX in 4-series leukotrienes (LT) and lipoxins (LX). In specific situations, some LT are involved in signalling pathways driving inflammatory responses as well as vascular permeability, or present chemo-attractant and pro-inflammatory properties. LX are anti-inflammatory mediators at nanomolar concentrations. These bioactive eicosanoids are described as being able to stimulate monocyte-derived macrophages to ingest and clear apoptotic neutrophils once mobilized on the site of inflammation and resolution.

LTB4, among its many roles, inhibits lymphocyte proliferation and promotes natural killer cell activity. 4-series LT also regulate production of pro-inflammatory cytokines; for example, LTB4 enhances production of TNF-alpha, IL-1, IL-6, IL-2 and IFN-gamma.

ARA can also be metabolized in epoxyeicosatrienoic (EET) and hydroxyeicosatetraenoic (HETE) acids by CYT P450. These metabolites possess anti-inflammatory properties, by regulating for example for some of them, diverse signalling pathways pertinent to platelet aggregation, inflammation and cellular injury.

Thus, ARA acid gives rise to a range of mediators which have opposing effects to one another, so the overall physiological effect will be governed by the concentration of those mediators, the timing of their production and the sensitivities of target cells to their effects.

\subsection{EPA and DHA as precursors of pro-resolving lipid mediators}

The same type of molecules is described for EPA and DHA metabolized to 3 -series of PG and TX, and to 5-series LT, which can competitively inhibit the release and metabolism of ARA, thereby reducing the body's inflammatory response and protecting from immune-mediated damage. 3-series PG are described as anti-inflammatory or less inflammatory than 2series PG issued from ARA. In the same way, 5-series LT are described as anti-inflammatory or less inflammatory than 4series LT.

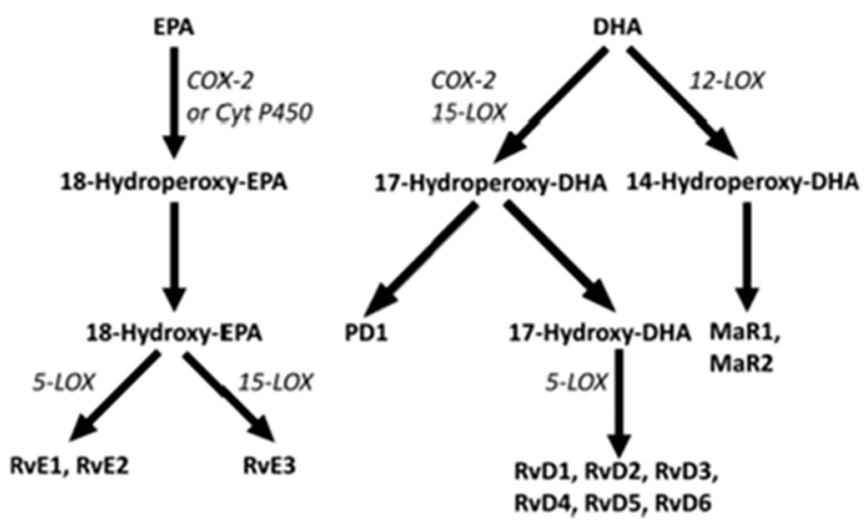

Fig. 3. Overview of the pathways of specialized pro-resolving mediators' synthesis from EPA and DHA. COX: cyclooxygenase; CYT P450: cytochrome P450 enzymes; DHA: docosahexaenoic acid; EPA: eicosapentaenoic acid; LOX: lipoxygenase; MaR: maresin: PD: protectin D; Rv: resolvin. Modified from Calder (2017) with permission from Portland Press.

CYT P450 also metabolize EPA into series of related biologically active mediators.

In addition to these metabolites, new endogenous bioactive derivatives from EPA and DHA have been recently described. These metabolites include resolvins produced from EPA (RvE) and DHA (RvD), and protectins (PD) and maresins (MaR) produced from DHA (Gilroy and Bishop-Bailey, 2019) via the COX and LOX pathways (Fig. 3). The powerful biological effects of Rv, MaR and PD would be at the basis of the antiinflammatory effects described for n-3 PUFAs.

These lipid mediators have indeed been described as playing a central role in the resolution of the inflammation. Duffney et al. (2018) define the resolution phase of a normal inflammatory response as being characterized by lipid mediator "class switching," in which cells downregulate enzymes responsible for the production of pro-inflammatory lipids, such as PG and LT, while upregulating enzymes responsible for the production of specialised pro-resolving lipid mediators. These mediators are unique in that they exert pro-resolving and anti-inflammatory effects without suppressing the immune response.

The biological effects of Rv, PD and MaR have been extensively studied in vitro as well as in vivo models of inflammation, and have been described as anti-inflammatory and inflammation resolving (Calder, 2017). RvE1, RvD1 and PD1 are described as inhibiting neutrophil migration and preventing neutrophil infiltration into inflammation sites. RvD1 inhibits the production of IL-1beta (a pro-inflammatory cytokine) and PD1 inhibits the production of TNF-alpha (involved in the inflammatory reaction) and IL-1beta (Caughey et al., 1996; Wada et al., 2007).

Regarding the roles of Rv, MaR and PD on the immune T and B cells, Duffney et al. (2018) reported that the production of IFN-gamma and TNF-alpha by specific subtypes of T cells is reduced by PD1. Recently, it has been shown that RvD1, $\mathrm{RvD} 2$, and MaR1 could similarly block the production of IFNgamma and TNF-alpha by $\mathrm{T}$ cells.

RvD1, RvD2, and MaR1 are also described as suppressing the differentiation of naive $T$ cells into specific subtypes while 
increasing the production of regulatory $\mathrm{T}$ cells known for their ability to produce pro-resolution factors to control inflammation such as transforming growth factor (TGF)-beta.

Concerning the $\mathrm{B}$ cell function, this one can be augmented, by a supplementation with the specialised pro-resolving lipid mediators or their precursor PUFA, in response to an increased antibody production, altered B cell cytokine production, skewed B cell differentiation/lineage, and limited spontaneous IgE class switching implicated in allergic asthma.

The discovery of these specialized pro-resolving lipid mediators, that actively promote the resolution of inflammation, has opened new avenues for the treatment of chronic inflammatory diseases, and has made them very promising therapeutic candidates, as reviewed by Duffney et al. (2018).

In their review, Gilroy and Bishop-Bailey (2019) also explain that one of the broader immunomodulatory properties of RvE1 is its ability to inhibit the accumulation of neutrophil and dendritic cells at sites of inflammation. Other actions of RvE1 include the inhibition of the biosynthesis of proinflammatory chemokine and cytokines, and the suppression of pro-inflammatory mechanisms while enhancing pro-resolution pathways.

RvD1 and D2 are described as also having both antiinflammatory and pro-resolution properties by blocking neutrophil infiltration, while also enhancing macrophage efferocytosis of apoptotic bodies.

PD1 is described as inhibiting the Toll-like receptors (TLR)-mediated activation (TLR are among the receptors involved in the first line of the actors deployed by the immune system against infection) while suppressing inflammatory cytokines and pro-inflammatory lipid mediator synthesis.

Concerning maresins, such as MaR1 and MaR2, a number of data are still missing.

In addition to the n-3 PUFA strongly implicated in the resolution of inflammation, Innes and Calder (2018) have recently proposed a complete review of the involvement of n-6 PUFA in the inflammatory response.

The authors recall that $n-6$ PUFA are described as being able to inhibit the anti-inflammatory and inflammation resolving effects of EPA and DHA because it is commonly believed that increasing dietary intake of ARA or its precursor LA will increase inflammation.

However, Innes and Calder report on studies in healthy human adults that have found that increased intake of ARA or LA does not increase the concentrations of many inflammatory markers, epidemiological studies even suggesting that ARA and LA may be linked to reduced inflammation.

\section{Dietary fatty acids as modulators of the immune cell function}

Modulation of the fatty acid composition of immune cell membranes under the influence of diet may have an impact on the activity of the immune cells, disrupting the immune functions (Calder, 2011; Kim et al., 2013).

Indeed, cell membrane fatty acids influence fundamental properties of the plasma membrane, notably membrane fluidity. Any modification of this fluidity or membrane order will have repercussions on the physical properties of these membranes, particularly within specific regions such as lipid rafts.

Since cell membranes serve as reservoirs for the formation of products with profound immune effects, these changes are not without consequences.

Thus, data obtained in humans have shown that the lipid content of our diet can modulate the immune response. For example, it has been observed that the proliferation of peripheral blood mononuclear cells cultured in the presence of specific mitogens was increased or even doubled in healthy women fed a diet for 40 days with a reduced fat content of 41 to 26 or $31 \%$ of total energy intake (Kelley et al., 1992a). In another study, conducted in healthy humans, it was shown that a decrease in diet lipid content of 30 to $25 \%$ of total energy intake induced a significant increase in the number of circulating $\mathrm{B}$ and $\mathrm{T}$ lymphocytes after 11 weeks, as well as an increase in their proliferation measured in vitro (Kelley, 2001). Similar data were obtained for lymphocyte proliferation (increased) and secretion (decreased) measured in vitro of IL-1 and TNF in elderly subjects whose lipid intakes were reduced by $36-27 \%$ of total energy intake for 6 months (Meydani et al., 1993). The results of two other studies conducted in elderly confirm the beneficial impact of a reduction in the lipid content of about $10 \%$ of the total energy intake with the demonstration of an increase in the proliferation capacity (Rasmussen et al., 1994) as well as an increased activity (Barone et al., 1989) of NK lymphocytes.

In his review about the impacts of the Western diet on immunity, Myles (2014) summarized the immunologic impacts of the different classes of dietary fatty acids. In addition to being characterized by an over consumption of refined sugars and salt, the Western diet is also characterized by its richness in SFA, n-6 PUFA and poverty in n-3 PUFA.

\subsection{SFA}

SFA are considered to be the most inflammatory compared to other fatty acid families.

They are described as capable of activating TLR-mediated pro-inflammatory signalling pathways in some immune cells such as macrophages and monocytes (Huang et al., 2012). TLR are involved in the detection of invading pathogens to evaluate if it is bacterial, viral and fungal. Then they activate the innate immune responses for host defence. Numerous studies with cells in culture and in animal models of mutated or deleted TLR4 or TLR2 subsequently demonstrated that SFA indeed can activate TLR4- and TLR2-mediated pro-inflammatory signalling pathway leading to expression of proinflammatory marker gene products (Lee et al., 2001; Lee et al., 2004; Wong et al., 2009).

As explained by Myles (2014), TLR4 senses bacteria, by binding the lipolysaccharide (LPS) of the bacteria, which contains SFA, mostly stearic and palmitic acids. This suggests that TLR4 can generate inappropriate signalling when exposed to certain SFA if in too great of frequency, amount, or homogeneity rather than in a more biological balance and dosage. As a result, this signalling anomaly may induce an inappropriate immune response in the case where SFA may be perceived as a bacterial invader. 


\subsection{N-6 PUFA}

Concerning the n-6 PUFA, the growth and development of lymphoid tissues and the structural and functional integrity of $\mathrm{T}$ and $\mathrm{B}$ lymphocytes are described as sensitive to their level, notably ARA and LA intakes, any deficiency in these PUFA may induce a loss of functional integrity of different cell types (monocytes, macrophages, neutrophils) (Guadarrama-Lopez et al., 2014).

With regard to LA, Blair et al. (1993) showed that a variation in its content between 3 and $8.3 \%$ of the total energy intake was accompanied by a variation in urinary PGE2 content, which is increased in women who consumed a diet richer in LA.

For ARA, it has been shown that supplementation at a rate of $1.5 \mathrm{~g}$ /day for 50 days in young healthy male subjects did not affect different parameters of the immune response; at the same time, the number of circulating neutrophils was increased by a factor of 4 (Kelley et al., 1997; Kelley et al., 1998a). Finally, in vitro data have shown the regulatory effect of ARA metabolites on the development and function of immunity cells including thymocyte growth and differentiation, $\mathrm{T}$ cell proliferation and migration, macrophage regulation, and pro-inflammatory cytokine production. Experimental data have also shown a direct relationship between the ARA content of phospholipids in immune cells and the ability of these cells to produce PGE2, such that this synthesis is increased when animals receive a diet enriched in ARA (Peterson et al., 1998), and decreased when animals are fed a diet enriched in EPA and DHA (Chapkin et al., 1991).

\subsection{N-3 PUFA}

As mentioned by Teague et al. (2016), EPA and DHA are bioactive molecules with great potential for manipulating the immune system, being able to influence both innate and adaptive immunity. N-3 PUFA have pleiotropic effects based on molecular mechanisms including lowering of ARA levels, and thereby downstream of pro-inflammatory mediators, and generation of specialized pro-resolving lipid mediators (Whelan et al., 2016).

Among the different dietary fatty acid classes, n-3 PUFA are described as having major anti-inflammatory effects, notably in the case of different physiopathological conditions with inflammatory components such as atherosclerosis and cardiovascular disease, inflammatory bowel, and allergic diseases. (Calder, 2001, 2017; Myles, 2014).

The existing data to date on the impact of n-3 PUFA on immune function mainly concern n-3 long-chain PUFA, EPA and DHA, and few their metabolic precursor ALA.

Kelley et al. (1991) studied for 8 weeks the effects of adding linseed oil to the diet of 10 healthy men (aged 2137 years) in order to increase their level of ALA intake from 1 to $18 \mathrm{~g} /$ day. This diet has resulted in increased levels of ALA, EPA and DHA in the lipids contained in peripheral mononuclear blood cells (Kelley et al., 1993), and decreased proliferation of these same cells in response to different recall antigens (Kelley et al., 1991). Overall, ALA tended to suppress some of indices of cell-mediated immunity (T-cell functions) without affecting any of the indices of humoral immunity (Bcell functions) tested.
In another study, healthy subjects (aged 24-44 years) were fed a diet containing flaxseed oil versus sunflower oil for 4 weeks, with lipids accounting for $30 \%$ of the total energy intake in both diets and the ALA intake level being 13.7 and $1.1 \mathrm{~g}$ respectively (Caughey et al., 1996). The diet enriched with ALA (linseed oil) reduced the production of proinflammatory cytokines (TNF-alpha, IL-1) measured in vitro.

Baker et al. (2016) recently reviewed the few controlled intervention studies investigating the effects of increased ALA consumption - from 2 to $20 \mathrm{~g}$ per day, for 8 to 24 weeks - on immune function other than inflammation have been conducted in adult human subjects.

Baker and co-authors quote, for example, Wallace et al. (2003) who reported, in a randomised, placebo-controlled, double-blind, parallel study in 40 healthy males (aged 1839 years) randomised to receive placebo or $3.5 \mathrm{~g}$ ALA per day or $0.44,0.94$ or $1.9 \mathrm{~g} /$ day $(\mathrm{EPA}+\mathrm{DHA})$ for 12 weeks, no effect of ALA on the functional activity of monocytes and lymphocytes. On the contrary, the two higher doses of $\mathrm{EPA}+\mathrm{DHA}$ resulted in a significant decrease in IL-6 production by stimulated mononuclear cells.

In a placebo-controlled, double-blind, parallel study in 150 healthy men and women (aged 25-72 years) randomly assigned to placebo, 4.5 or $9.5 \mathrm{~g} \mathrm{ALA} / \mathrm{d}, 0.77$ or $1.7 \mathrm{~g}$ $\mathrm{EPA}+\mathrm{DHA} / \mathrm{d}$ for 6 months, Kew et al. (2003b) observed no effect of ALA (and EPA + DHA) on the functional activity of neutrophils, monocytes, or lymphocytes.

Thies et al. (2001a) assessed in a randomized, placebocontrolled, double-blind and parallel assay in healthy subjects (aged 55-75 years), the effects of a 12-week dietary supplementation with moderate levels of ALA $(2 \mathrm{~g} / \mathrm{d})$ versus other PUFA (gamma-linolenic acid (GLA), ARA, DHA or $\mathrm{EPA}+\mathrm{DHA}$ ) on the proliferation of mitogen-stimulated human peripheral blood mononuclear cells (PBMC) and on the production of cytokines by those cells. Although some of the other PUFA (GLA and EPA + DHA) caused a significant decrease (up to $65 \%$ ) in lymphocyte proliferation, the treatment with ALA not affected the production of IL-2 or IFN-gamma by PBMC, or the number or proportion of T or B lymphocytes, helper or cytotoxic $\mathrm{T}$ lymphocytes or memory helper $\mathrm{T}$ lymphocytes in the circulation. The same type of study was conducted to assess the effects of the same dietary supplementations on the NK cell activity of human PBMC. A treatment with ALA had no effect on the NK cell activity in healthy subjects (Thies et al., 2001b).

As a conclusion, Baker et al. (2016) acted that except very high intakes of ALA suppressing some aspects of immune function, it seems that moderate-high intakes of ALA (2 to $9.5 \mathrm{~g} / \mathrm{d}$ ) for at least 20 weeks may have no impact on the immune function.

Similarly, different authors have investigated the effects of EPA and DHA supplementation. Thus, in vitro data showed that supplementation of the culture medium with EPA and DHA inhibited the production of different pro-inflammatory cytokines (TNF-alpha, IL-1, IL-6) for different cell types (Babcock et al., 2002; Zhao et al., 2004).

In humans, the impact of fish consumption as a source of EPA and DHA has also been studied. A study testing the effects of consuming 120 to $188 \mathrm{~g}$ of fish per day (or $1.23 \mathrm{~g}$ /day of EPA + DHA) for 6 months in elderly subjects showed a reduction in lymphocyte proliferation, as well as in the 
production of different pro-inflammatory cytokines measured in vitro (IL-1, IL-6 and TNF-alpha (Meydani et al., 1993). On the other hand, a study conducted in healthy subjects consuming $500 \mathrm{~g}$ salmon per day $(2.3 \mathrm{~g}$ EPA and $3.6 \mathrm{~g}$ DHA/day) for 40 days, versus a control diet, did not show any effects on different parameters of the immune response (Kelley et al., 1992b).

A number of studies have also evaluated the effects of fish oil-based supplementation, with tested amounts ranging from 2 to $30 \mathrm{~g}$ per day (or 0.55 to $8 \mathrm{~g} /$ day of EPA + DHA), over periods ranging from 4 to 52 weeks (Kelley, 2001). Most studies have shown a 25 to $75 \%$ decrease in the secretion of these cytokines measured in vitro in response to EPA and DHA supplementation of $2.4 \mathrm{~g} /$ day or more for at least 4 weeks in healthy subjects (Kelley et al., 1998b; Kelley et al., 1999; Babcock et al., 2002). Conversely, there does not appear to be any effects on these cytokines for lower EPA + DHA supplementation (Mølvig et al., 1991; Cooper et al., 1993; Schmidt et al., 1996).

Finally, other data have shown the effect of DHA alone $(6 \mathrm{~g} /$ day for 12 weeks $)$ with a decrease in the number of circulating neutrophils, a decrease in the production of cytokines (IL-1 beta and TNF-alpha) and pro-inflammatory eicosanoids from ARA (PGE2 and LTB4) of around 25 to $70 \%$. In contrast, DHA supplementation has not reduced the proliferation of $\mathrm{B}$ and $\mathrm{T}$ lymphocytes suggesting that DHA can be used specifically to inhibit inflammatory responses without inhibiting immune responses involving $\mathrm{B}$ and $\mathrm{T}$ lymphocytes (Kelley et al., 1999).

Recently, Whelan et al. (2016) reviewed from pre-clinical models the emerging evidence that n-3 PUFA (from fish oil) could target the function of $\mathrm{B}$ cells which are involved in inflammatory and humoral immune responses. Existing data suggest that n-3 PUFA may modify B cell antigen presentation, cytokine production and antibody generation. Several mechanisms of action are mentioned, including involvement of Th2 cytokines, enhanced production of specialized pro-resolving lipid mediators, and targeting protein lateral organization in lipid microdomains.

The impact of an intake of EPA + DHA in the form of fish oil has also been investigated on the synthesis of resolvins (Calder, 2017). Thus, in rodents, an increased synthesis of resolvins was observed in animals fed a fish oil supplemented diet. In humans, significant concentrations of RvE1 and RvD1 were observed in the plasma of healthy volunteers subjected to 3 weeks of nutritional supplementation with fish oil.

Regarding the effects of EPA and DHA on the immune cell functional responses, it is difficult to construct a dose-response relationship from the existing literature. According to Calder (2017), some of the studies that fail to show an effect of n-3 PUFA on cytokine production have provided less than $2 \mathrm{~g}$ of EPA + DHA per day, which may be an insufficient dose. Others have indicated reduction in the production of inflammatory mediators with a minimum of $1.2 \mathrm{~g}$ per day of supplementation with EPA + DHA for 6 weeks (Kelley, 2001). Therefore, further studies are needed to establish adequate intake levels of n-3 PUFA.

\section{Conclusion}

Taken together, these data show an effect of dietary fatty acids on immune and inflammatory responses. However, the contradictory effects observed between studies may be explained by differences in lipid contents and fatty acid composition of the different diets tested, supplementation times, as well as the populations tested (age and health status).

There are many data on the effects of EPA and DHA diet supplementation on the functionality of immune cells. Among these, mention is made of the ability of these PUFA to decrease the production of pro-inflammatory eicosanoids from ARA, or pro-inflammatory cytokines, which can reduce the inflammatory component of the immune response.

Progress in understanding the mechanisms of action of n-3 PUFA has been made over the past 10 years, including the identification of novel bioactive lipid mediators with major inflammatory properties.

These specialized pro-resolving lipid mediators, associated with eicosanoids, are key signalling molecules in the immune functions, notably the resolution of inflammation, by playing a pivotal role in regulating the inflammatory profile and promoting return to homeostasis. But it seems that the interaction of n-3 and n-6 PUFA and their lipid mediator derivatives in the context of immunity and inflammation is complex and still not properly understood.

\section{References}

Arterburn LM, Hall EB, Oken H. 2006. Distribution, interconversion, and dose response of n-3 fatty acids in humans. Am J Clin Nutr 83: $1467 \mathrm{~S}-1476 \mathrm{~S}$.

Babcock TA, Novak T, Ong E, Jho DH, Helton WS, Espat NJ. 2002. Modulation of lipopolysaccharide-stimulated macrophage tumor necrosis factor-alpha production by omega-3 fatty acid is associated with differential cyclooxygenase- 2 protein expression and is independent of interleukin-10. J Surg Res 107: 135-139.

Baker EJ, Miles EA, Burdge GC, Yaqoob P, Calder PC. 2016. Metabolism and functional effects of plant-derived omega-3 fatty acids in humans. Prog Lipid Res 64: 30-56.

Barone J, Hebert JR, Reddy MM. 1989. Dietary fat and natural-killercell activity. Am J Clin Nutr 50: 861-867.

Blair IA, Prakash C, Phillips MA, Dougherty RM, Iacono JM. 1993. Dietary modification of omega 6 fatty acid intake and its effect on urinary eicosanoid excretion. Am J Clin Nutr 57: 154-160.

Blanchard H, Pédrono F, Boulier-Monthéan N, Catheline D, Rioux V, Legrand P. 2013. Comparative effects of well-balanced diets enriched in $\alpha$-linolenic or linoleic acids on LC-PUFA metabolism in rat tissues. Prostaglandins Leukot Essent Fatty Acids 88: 383-389.

Boissonneault GA. 2008. Dietary fat, immunity, and inflammatory disease. In: Chow CK, ed. Fatty acids in foods and their health implications. 3rd ed. Boca Raton: CRC Press.

Burdge G, Calder P. 2006. Dietary alpha-linolenic acid and healthrelated outcomes: A metabolic perspective. Nutr Res Rev 19:26-52.

Burdge GC, Wootton SA. 2002. Conversion of alpha-linolenic acid to eicosapentaenoic, docosapentaenoic and docosahexaenoic acids in young women. Br J Nutr 88: 411-420. 
Calder PC. 2001. N-3 polyunsaturated fatty acids, inflammation and immunity: Pouring oil on troubled waters or another fishy tale? Nutr Res 21: 309-341.

Calder PC. 2011. Fatty acids and inflammation: The cutting edge between food and pharma. Eur J Pharmacol 668: S50-S58.

Calder PC. 2013. N-3 fatty acids, inflammation and immunity: New mechanisms to explain old actions. Proceed Nutr Soc 72: 326336.

Calder PC. 2015. Marine omega-3 fatty acids and inflammatory processes: Effects, mechanisms and clinical relevance. Biochim Biophys Acta 1851: 469-484.

Calder PC. 2017. Omega-3 fatty acids and inflammatory processes: From molecules to man. Biochem Soc Trans 45: 1105-1115.

Calder PC, Bond JA, Harvey DJ, Gordon S, Newsholme EA. 1990. Uptake and incorporation of saturated and unsaturated fatty acids into macrophage lipids and their effect upon macrophage adhesion and phagocytosis. Biochem J 269: 807-814.

Calder PC, Bevan SJ, Newsholme EA. 1992. The inhibition of Tlymphocyte proliferation by fatty acids is via an eicosanoidindependent mechanism. Immunology 75: 108-115.

Calder PC, Yaqoob P, Harvey DJ, Watts A, Newsholme EA. 1994. Incorporation of fatty acids by concanavalin A-stimulated lymphocytes and the effect on fatty acid composition and membrane fluidity. Biochem J 300(Pt 2): 509-518.

Caughey GE, Mantzioris E, Gibson RA, Cleland LG, James MJ. 1996. The effect on human tumor necrosis factor $\alpha$ and interleukin $1 \beta$ production of diets enriched in n-3 fatty acids from vegetable oil or fish oil. Am J Clin Nutr 63: 116-122.

Chapkin RS, Akoh CC, Miller CC. 1991. Influence of dietary n-3 fatty acids on macrophage glycerophospholipid molecular species and peptidoleukotriene synthesis. J Lipid Res 32: 1205-1213.

Cooper AL, Gibbons L, Horan MA, Little RA, Rothwell NJ. 1993. Effect of dietary fish oil supplementation on fever and cytokine production in human volunteers. Clin Nutr 12: 321-328.

Duffney PF, Falsetta ML, Rackow AR, Thatcher TH, Phipps RP, Sime PJ. 2018. Key roles for lipid mediators in the adaptive immune response. J Clin Invest 128: 2724-2731.

Faber J, Berkhout M, Vos AP, et al. 2011. Supplementation with a fish oil-enriched, high-protein medical food leads to rapid incorporation of EPA into white blood cells and modulates immune responses within one week in healthy men and women. $J$ Nutr 141: 964-970.

Gilroy DW, Bishop-Bailey D. 2019. Lipid mediators in immune regulation and resolution. Br J Pharmacol 176: 1009-1023.

Guadarrama-Lopez AL, Valdes-Ramos R, Martinez-Carrillo BE. 2014. Type 2 diabetes, PUFAs, and vitamin D: Their relation to inflammation. J Immunol Res 2014: 860703.

Harnack K, Andersen G, Somoza V. 2009. Quantitation of alphalinolenic acid elongation to eicosapentaenoic and docosahexaenoic acid as affected by the ratio of $\mathrm{n} 6 / \mathrm{n} 3$ fatty acids. Nutr Metabol 6: 8 .

Huang S, Rutkowsky JM, Snodgrass RG, et al. 2012. Saturated fatty acids activate TLR-mediated proinflammatory signaling pathways. J Lipid Res 53: 2002-2013.

Hubler MJ, Kennedy AJ. 2016. Role of lipids in the metabolism and activation of immune cells. J Nutr Biochem 34: 1-7.

Innes JK, Calder PC. 2018. Omega-6 fatty acids and inflammation. Prostaglandins Leukot Essent Fatty Acids 132: 41-48.

Kalinski P. 2012. Regulation of immune responses by prostaglandin E2. J Immunol 188: 21-28.

Kelley DS. 2001. Modulation of human immune and inflammatory responses by dietary fatty acids. Nutrition 17: 669-673.
Kelley DS, Branch LB, Love JE, Taylor PC, Rivera YM, Iacono JM. 1991. Dietary alpha-linolenic acid and immunocompetence in humans. Am J Clin Nutr 53: 40-46.

Kelley DS, Dougherty RM, Branch LB, Taylor PC, Iacono JM. 1992a. Concentration of dietary N-6 polyunsaturated fatty acids and the human immune status. Clin Immunol Immunopathol 62: 240-244.

Kelley DS, Nelson GJ, Branch LB, Taylor PC, Rivera YM, Schmidt PC. 1992b. Salmon diet and human immune status. Eur J Clin Nutr 46: 397-404.

Kelley DS, Nelson GJ, Love JE, et al. 1993. Dietary alpha-linolenic acid alters tissue fatty acid composition, but not blood lipids, lipoproteins or coagulation status in humans. Lipids 28: 533-537.

Kelley DS, Taylor PC, Nelson GJ, Schmidt PC, Mackey BE, Kyle D. 1997. Effects of dietary arachidonic acid on human immune response. Lipids 32: 449-456.

Kelley DS, Taylor PC, Nelson GJ, Mackey BE. 1998a. Arachidonic acid supplementation enhances synthesis of eicosanoids without suppressing immune functions in young healthy men. Lipids 33: $125-130$.

Kelley DS, Taylor PC, Nelson GJ, Mackey BE. 1998b. Dietary docosahexaenoic acid and immunocompetence in young healthy men. Lipids 33: 559-566.

Kelley DS, Taylor PC, Nelson GJ, et al. 1999. Docosahexaenoic acid ingestion inhibits natural killer cell activity and production of inflammatory mediators in young healthy men. Lipids 34: 317-324.

Kew S, Banerjee T, Minihane AM, et al. 2003a. Relation between the fatty acid composition of peripheral blood mononuclear cells and measures of immune cell function in healthy, free-living subjects aged 25-72 y. Am J Clin Nutr 77: 1278-1286.

Kew S, Banerjee T, Minihane AM, et al. 2003b. Lack of effect of foods enriched with plant- or marine-derived n-3 fatty acids on human immune function. Am J Clin Nutr 77: 1287-1295.

Kim K, Jung N, Lee K, et al. 2013. Dietary omega-3 polyunsaturated fatty acids attenuate hepatic ischemia/reperfusion injury in rats by modulating toll-like receptor recruitment into lipid rafts. Clin Nutr 32: 855-862.

Kinsella JE, Lokesh B, Broughton S, Whelan J. 1990. Dietary polyunsaturated fatty acids and eicosanoids: Potential effects on the modulation of inflammatory and immune cells: An overview. Nutrition 6: 24-44.

Lee JY, Sohn KH, Rhee SH, Hwang D. 2001. Saturated fatty acids, but not unsaturated fatty acids, induce the expression of cyclooxygenase-2 mediated through Toll-like receptor 4. J Biol Chem 276: 16683-16689.

Lee JY, Zhao L, Youn HS et al. 2004. Saturated fatty acid activates but polyunsaturated fatty acid inhibits Toll-like receptor 2 dimerized with Toll-like receptor 6 or 1. J Biol Chem 279: 16971-16979.

Lewis RA, Austen KF, Soberman RJ. 1990. Leukotrienes and other products of the 5-lipoxygenase pathway: Biochemistry and relation to pathobiology in human diseases. $N$ Engl J Med 323: 645-655.

Maggini S, Pierre A, Calder PC. 2018. Immune function and micronutrient requirements change over the life course. Nutrients 10: 1531 .

Mantzioris E, James MJ, Gibson RA, Cleland LG. 1994. Dietary substitution with an alpha-linolenic acid-rich vegetable oil increases eicosapentaenoic acid concentrations in tissues. $\mathrm{Am} \mathrm{J}$ Clin Nutr 59: 1304-1309.

Marshall LA, Johnston PV. 1983. The effect of dietary alpha-linolenic acid in the rat on fatty acid profiles of immunocompetent cell populations. Lipids 18: 737-742. 
Maskrey BH, Megson IL, Rossi AG, Whitfield PD. 2013. Emerging importance of omega-3 fatty acids in the innate immune response: Molecular mechanisms and lipidomic strategies for their analysis. Mol Nutr Food Res 57: 1390-1400.

Meydani SN, Lichtenstein AH, Cornwall S, et al. 1993. Immunologic effects of national cholesterol education panel step-2 diets with and without fish-derived N-3 fatty acid enrichment. J Clin Invest 92: 105-113.

Miles EA, Banerjee T, Calder PC. 2004. The influence of different combinations of gamma-linolenic, stearidonic and eicosapentaenoic acids on the fatty acid composition of blood lipids and mononuclear cells in human volunteers. Prostaglandins Leukot Essent Fatty Acids 70: 529-538.

Mølvig J, Pociot F, Worsaae H, et al. 1991. Dietary supplementation with omega-3-polyunsaturated fatty acids decreases mononuclear cell proliferation and interleukin-1 beta content but not monokine secretion in healthy and insulin-dependent diabetic individuals. Scand J Immunol 34: 399-410.

Myles IA. 2014. Fast food fever: Reviewing the impacts of the Western diet on immunity. Nutr J 13: 61.

Peterson LD, Jeffery NM, Thies F, Sanderson P, Newsholme EA, Calder PC. 1998. Eicosapentaenoic and docosahexaenoic acids alter rat spleen leukocyte fatty acid composition and prostaglandin E2 production but have different effects on lymphocyte functions and cell-mediated immunity. Lipids 33: 171-180.

Rasmussen LB, Kiens B, Pedersen BK, Richter EA. 1994. Effect of diet and plasma fatty acid composition on immune status in elderly men. Am J Clin Nutr 59: 572-577.

Schmidt EB, Varming K, Møller JM, Bülow Pedersen I, Madsen P, Dyerberg J. 1996. No effect of a very low dose of n-3 fatty acids on monocyte function in healthy humans. Scand J Clin Lab Invest 56: 87-92.

Simopoulos AP. 2011. Importance of the omega-6/omega-3 balance in health and disease: Evolutionary aspects of diet. World Rev Nutr Diet 102: 10-21.

Sioen I, van Lieshout L, Eilander A, et al. 2017. Systematic review on $\mathrm{N}-3$ and N-6 polyunsaturated fatty acid intake in European countries in light of the current recommendations-Focus on specific population groups. Ann Nutr Metab 70: 39-50.

Teague H, Harris M, Whelan J, Comstock SS, Fenton JI, Shaikh SR. 2016. Short-term consumption of n-3 PUFAs increases murine IL-5 levels, but IL- 5 is not the mechanistic link between n-3 fatty acids and changes in B-cell populations. J Nutr Biochem 28: 30-36.

Thies F, Nebe-von-Caron G, Powell JR, Yaqoob P, Newsholme EA, Calder PC. 2001a. Dietary supplementation with gamma-linolenic acid or fish oil decreases T lymphocyte proliferation in healthy older humans. J Nutr 131: 1918-1927.

Thies F, Nebe-von-Caron G, Powell JR, Yaqoob P, Newsholme EA, Calder PC. 2001b. Dietary supplementation with eicosapentaenoic acid, but not with other long-chain n-3 or n-6 polyunsaturated fatty acids, decreases natural killer cell activity in healthy subjects aged $>55$ y. Am J Clin Nutr 73: 539-48.

Tilley SL, Coffman TM, Koller BH. 2001. Mixed messages: Modulation of inflammation and immune responses by prostaglandins and thromboxanes. J Clin Invest 108: 15-23.

Tiwari RK, Clandinin MT, Cinader B. 1986. Effect of high/low dietary linoleic acid feeding on mouse splenocytes: Modulation by age and influence of genetic variability. Nutr Res 6: 13791387.

Tiwari RK, Clandinin MT, Cinader B. 1987. Effect of high polyunsaturated fat diets on the composition of $\mathrm{B}$ cell and $\mathrm{T}$ cell membrane lipids. Nutr Res 7: 489-498.

Wada M, DeLong CJ, Hong YH, et al. 2007. Enzymes and receptors of prostaglandin pathways with arachidonic acid-derived versus eicosapentaenoic acid-derived substrates and products. $J$ Biol Chem 282: 22254-22266.

Wall R, Ross RP, Fitzgerald GF, Stanton C. 2010. Fatty acids from fish: The anti-inflammatory potential of long-chain omega-3 fatty acids. Nutr Rev 68: 280-289.

Wallace FA, Miles EA, Calder PC. 2003. Comparison of the effects of linseed oil and different doses of fish oil on mononuclear cell function in healthy human subjects. $\mathrm{Br} J \mathrm{Nutr}$ 89: 679-689.

Whelan J, Gowdy KM, Shaikh SR. 2016. N-3 polyunsaturated fatty acids modulate $\mathrm{B}$ cell activity in pre-clinical models: Implications for the immune response to infections. Eur J Pharmacol 785: 10-17.

Wong SW, Kwon MJ, Choi AM, Kim HP, Nakahira K, Hwang DH. 2009. Fatty acids modulate Toll-like receptor 4 activation through regulation of receptor dimerization and recruitment into lipid rafts in a reactive oxygen species-dependent manner. $J$ Biol Chem 284: 27384-27392.

Yaqoob P, Pala HS, Cortina-Borja M, Newsholme EA, Calder PC. 2000. Encapsulated fish oil enriched in alpha- tocopherol alters plasma phospholipid and mononuclear cell fatty acid compositions but not mononuclear cell functions. Eur J Clin Invest 30: 260-274.

Zhao Y, Joshi-Barve S, Barve S, Chen LH. 2004. Eicosapentaenoic acid prevents LPS-induced TNF-alpha expression by preventing NF-kappaB activation. J Am Coll Nutr 23: 71-78.

Cite this article as: Buaud B. 2020. How fats we eat modulate our immunity?. OCL 27: 22. 